\title{
Absehen, Ablesen oder Lippenlesen? Patienteninformation
}

\begin{abstract}
Absehen, Ablesen oder Lippenlesen? Sowohl in der Fachliteratur als auch in der Umgangssprache existieren genannte Begriffe parallel nebeneinander - weitere sind Mundablesen, Sprechlesen und Sprechsehen. Die Vielfalt der Begriffe trägt nicht unbedingt zur fachlichen Auseinandersetzung bei, wenn auch davon auszugehen ist, dass dem Fachmann klar ist, was gemeint ist. Bei Personen, die zunächst als Laien mit dem Thema in Kontakt kommen, als z.B. im späteren Lebensalter von einer Hörschädigung betroffene Menschen, führt es eher zur Verunsicherung.
\end{abstract}

Die Vielfalt der Begriffe hat mehrere Ursachen. Eine liegt beispielsweise in historischen (Fehl-)Auffassungen zur Leistungsfähigkeit des Absehens. So ging Graser in seiner Schrift „Der durch Gesicht und Tonsprache der Menschheit wiedergegebene Taubstumme" von 1829 von einem Mundalphabet aus und überschätzte damit die Möglichkeiten des Absehens bei weitem. Eine weitere Ursache liegt im unkritischen Übertragen des im englischsprachigen Raum benutzten Terminus „lip reading" ins Deutsche. Insbesondere in der deutschsprachigen medizinischen Fachliteratur hat sich in Folge dessen das Wort „Lippenlesen“ verankert, ohne sich zuvor tatsächlich mit dem Absehvorgang näher auseinanderzusetzen.

\section{Was spricht gegen ,-lesen“? \\ $\nabla$}

Das Hauptargument gegen Wortverbindungen mit "-lesen“ (also Lippenlesen, Mundablesen, Sprechlesen oder Sprachlesen) kann sein, dass es kein dem Buchstabenalphabet vergleichbares sichtbares Mundalphabet gibt. Außerdem ist ein Großteil der Bewegungen der Artikulationsorgane kaum oder nicht sichtbar. Hinzukommt, dass die Sprechbewegungen bei jedem einzelnen Unterschiede aufweisen. Ein genormtes Mundbild wie die gedruckte Schrift gibt es nicht. Die Schrift- sprache hat weitere Vorteile, die das Absehbild nicht liefern kann. Beispiele sind ihre Beständigkeit (bei Nichtverstehen kann ich wiederholt (nach)lesen), die Groß- und Kleinschreibung, die das Verstehen bzw. die Aufnahme unterstützen, oder Wortzwischenräume, Interpunktion und Untergliederungen in Absätze, die zusätzliche Gliederungshilfen bieten.

Beim Absehen hingegen hat der Absehende stets nur ein lückenhaftes, mehrdeutiges Absehbild zur Verfügung. Ein Vergleich mit bzw. eine Verbindung zum Lesen herzustellen, scheint daher wenig gerechtfertigt. Ebenso ist eine Einengung auf „Mund“ oder „Lippen“ - z.B. Mundablesen, Mundabsehen oder Lippenlesen nicht angebracht, da nachweislich vom Absehenden das gesamte Gesicht (also auch die Wangen, der Kieferwinkel und sogar der Kehlkopf) sowie die Mimik (und Gestik) herangezogen wird.

\section{Warum Absehen? \\ $\nabla$}

Auch wenn der Begriff „Absehen“ nicht den gesamten Perzeptionsvorgang erfasst, der beim Absehen von Lautsprache über den visuellen Kanal notwendig ist, scheint es derjenige zu sein, der den wissenschaftstheoretischen Anforderungen und zugleich einer allgemeinen Verständlichkeit am ehesten gerecht wird.

\section{Bedeutung des Absehens und beeinflussende Faktoren \\ $\nabla$}

Absehen hat trotz leistungsfähiger Hörhilfen (digitale Hörgeräte, Cochlea-Implantate) nichts an seiner Bedeutung eingebüßt. Es ist eine Fähigkeit, die durch intensives Üben gesteigert werden kann. Auch normal hörende Personen nutzen das Absehen, z.B. schaut man unter Störschallbedingungen dem Gesprächspartner auf dem Mund. Menschen mit Hörschädigung erreichen erfahrungsgemäß einen höhe- ren Kompetenzgrad, da sie darauf angewiesen sind und das Absehen bewusster einsetzen. Beeinflussend wirkt auch die Sprachkompetenz des Absehenden („man kann nur Wörter absehen, die man kennt“). Kinder mit angeborenen Hörschäden erwerben die Fähigkeit zum Absehen parallel zum Lautspracherwerb. Personen, die erst später von einem Hörverlust betroffen sind, können auf vorhandene Sprache zurückgreifen. Sie müssen lernen, durch bewusstes Absehen ihr eingeschränktes Hörvermögen auszugleichen, indem sie sich die Absehgestalten bewusst machen. Hilfreich können Absehkurse (z.B. angeboten durch den Deutschen Schwerhörigenbund oder die Volkshochschule) sein.

Das Absehen wird durch verschiedene Faktoren beeinflusst, z.B. organisatorischtechnische Bedingungen (wie Entfernung zwischen Absehenden und Sprecher, Beleuchtung, Lichtverhältnisse), sprachliches Material (z.B. 3- oder 4-silbige Wörter werden besser abgesehen als 1 - oder 2-silbige, da Kombinationsmöglichkeiten vorhanden sind; kurze, klare und vollständige Sätze sind besser abzusehen als längere und komplizierte syntaktische Strukturen), Bedingungen, die vom Sprecher ausgehen (wie Sprechweise und -tempo) oder Bedingungen, die durch Absehenden bedingt sind (Konzentriertheit, Ermüdung).

\section{Fazit}

Absehen ist der inhaltlich treffendste Begriff. Eine zu starke Einengung auf Wortverbindungen mit "-lesen“ oder „Mund-“" wird dem komplexen Vorgang nicht gerecht und ist zudem irreführend.

\section{Prof. Dr. Annette Leonhardt, München}

überreicht durch Thieme und 